\title{
CHOLESTASIS: MOLECULAR MECHANISM, PATHOGENESIS AND POSSIBLE MANAGEMENT.
}

\author{
BY \\ Basant Mahmoud Al-Botaty ${ }^{\text {a,*; }}$; Amany Ali Eissa Ahmed ${ }^{\text {a }}$; Rehab Kamel ${ }^{\text {a,b }}$ \\ FROM \\ ${ }^{a}$ Pharmacology and Toxicology Department, Faculty of Pharmacy, Helwan \\ University, Cairo, Egypt. \\ ${ }^{\mathrm{b}}$ Pharmacology and Toxicology Department, Faculty of Pharmacy, Ahram Canadian \\ University, Cairo, Egypt.
}

\begin{abstract}
Understanding the molecular mechanism of bile formation and the different pathways and pathogenesis of drug induced cholestasis provide different possibilities for treatment. This review summarizes the role of transport proteins in hepatic drug clearance and toxicity, the involvement of inflammatory mediators in cholestasis and the role of nuclear receptors in bile acid metabolism. Understanding nuclear receptor function can help in the development of nuclear receptor ligands for treatment of cholestasis.
\end{abstract}

\section{Keywords:}

Bile acids, nuclear receptors, inflammatory mediators, management.

Bile acids.

Bile is exclusively synthesized in the liver. Some of its constituents are bile acids, bile pigments (mainly bilirubin), cholesterol, endogenous metabolites, phospholipids and xenobiotics. Bile function include increasing $\mathrm{pH}$ in the duodenum, due to its slightly alkaline nature, which provide an optimum environment for the pancreatic enzymes to work (Chaudhuri, 2006).

Bile acids are amphipathic molecules derived from cholesterol. It represents a major pathway for hepatic cholesterol catabolism to finally produce amphipathic molecules that serve as powerful physiological detergents with the role of absorption and transportation of nutrients, vitamins and fats (Russell and Setchell, 1992; Chiang, 2004).

\section{Synthesis of bile acids.}

Bile acids are the major driving force of bile flow. Thus, it is essential to maintain the bile acid content to ensure adequate bile flow. Bile acids pool consists of primary bile acids (cholic acid (CA), chenodeoxycholic acid (CDCA)) and secondary bile acids (deoxycholic acid (DCA), and lithocholic acid (LCA)) (Russell and Setchell, 1992).

Primary bile acids are synthesized exclusively in the liver. Cholesterol $7 \alpha-$ hydroxylase (CYP7A1), a microsomal cytochrome p450 enzyme is responsible for the first and the rate-limiting step in the main pathway of converting cholesterol into $7 \alpha-$ hydroxycholesterol and finally to CA and CDCA whereas CYP27A1 is an enzyme involved in the alternative pathway (Myant and Mitropoulos, 1977). 
Secondary bile acids are obtained furtherly from primary bile acids by dehydroxylation (losing - $\mathrm{OH}$ group at $\mathrm{C} 7$ position) and deconjugation which is done by the action of intestinal bacteria, where CA is converted to DCA and CDCA is converted to LCA. DCA is reabsorbed back and enters the liver sinusoids but LCA is directly excreted via faeces (Chiang, 2002; Chiang, 2004).

\section{Enterohepatic circulation of bile acids.}

Bile acids are produced in the liver and released through the transporters of the hepatocytes into the bile and stored in the gallbladder. After each meal, the gall bladder contracts to secrete bile acids into the intestinal tract. The majority of the bile acids $(95 \%)$ are reabsorbed in the ileum, and transported back to the liver via the portal blood for re-excretion into the bile. This process is referred to as enterohepatic circulation of the bile acids (Meier, 1995).

Conjugated bile acids are secreted into the bile by active transport mechanism. If their concentrations in the hepatocytes exceed the capacity of the active transport, the liver spontaneously spill them over into the sinusoid blood. Bile acids in the blood circulation are often reabsorbed through the renal tubules in the kidney and are circulated back to the liver through systemic circulation. The bile acids pool is about 3 $\mathrm{g}$ which is recycled 4-12 times a day. The daily minute amount lost in the faeces $(0.2-$ $0.6 \mathrm{~g} / \mathrm{day}$ ) are replenished by de novo synthesis in the liver to maintain a constant bile acids pool (Chiang, 2009).

\section{Transportation of bile acids.}

Hepatocytes are polarized epithelial cells with basolateral (sinusoidal) and apical (canalicular) membrane domains. Hepatocytes uptake bile acids from the portal blood plasma through the basolateral membrane and excrete them at the canalicular membrane into the bile (Boyer, 1980). It is recognized that the biliary bile acids concentration is about 100 to 1000 fold higher than in the hepatocytes, thus active transport is required for transporting the bile acids against the gradient from the hepatocyte cell membrane into the bile duct cell membrane. This explains why the canalicular bile acids transport represent the rate-limiting step in bile formation (Meier, 1995).

The ATP-binding cassette (ABC) transporter family are responsible for transporting bile acids and other organic compounds across the canalicular membrane against their concentration gradients ( $\mathrm{Li}$ and Chiang, 2009). Correspondingly, two bile acid transporters, sodium-dependent taurocholate cotransporter (NTCP) and organic anion transporter proteins (OATPs) are responsible for basolateral bile acid transport into the hepatocytes (Meier and Stieger, 2002).

\section{Apical (canalicular) bile acid transporters (ATP- binding cassette(ABC) transporter family).}

Most of the solute transport-proteins belong to the superfamily of $\mathrm{ABC}$ transporters. The ABC transporters are synthesized in the endoplasmic reticulum followed by glycosylation in the trans-Golgi network and finally travelling through the subapical compartment to the canalicular membrane. One type of the canalicular ABC transporters which controls the transport of conjugated bile acids (CA and CDCA) is the bile salt export pump (BSEP) (Ortiz et al., 2004). 
Canalicular ABC transporters also include multidrug resistance associated proteins (MRP2) which are responsible for the transportation of bile acids, glucuronides and glutathione (Alrefai and Gill, 2007). MRP4 is a cotransporter of bile acids and glutathione. The presence of bile acids in the bile enhances the secretion of phospholipids and cholesterol into the bile, followed by passive inflow of water (Oude Elferink and Groen, 2000). Phospholipids are excreted by the multidrug resistance protein (MDR2) (MDR3 in humans) (Gonzales et al., 2004) (Figure 1).

When the bile acids concentration in a solution is within a critical value, called critical micellization concentration, the bile acids form mixed micelles by association with phospholipids and cholesterol. Such mixed micelles prevent the direct contact of the bile acids to the epithelial cells, which accounts for the lack of bile acids-induced injury in the biliary system and small intestine under physiological circumstances (Natalini et al., 2014). Mutations of the MDR2 gene lead to the formation of phosphatidylcholine-poor bile with a high bile acids concentration. The high content of bile acids in the absence of phospholipids hinders the formation of micelles rendering the bile toxic to the bile duct epithelium (Balistreri et al., 2005).

ATP binding cassette subfamily G member 5 and 8 also belongs to the family of ATPdependant $\mathrm{ABC}$ transporters and is responsible for the secretion of free cholesterol into the bile (Yu et al., 2002).

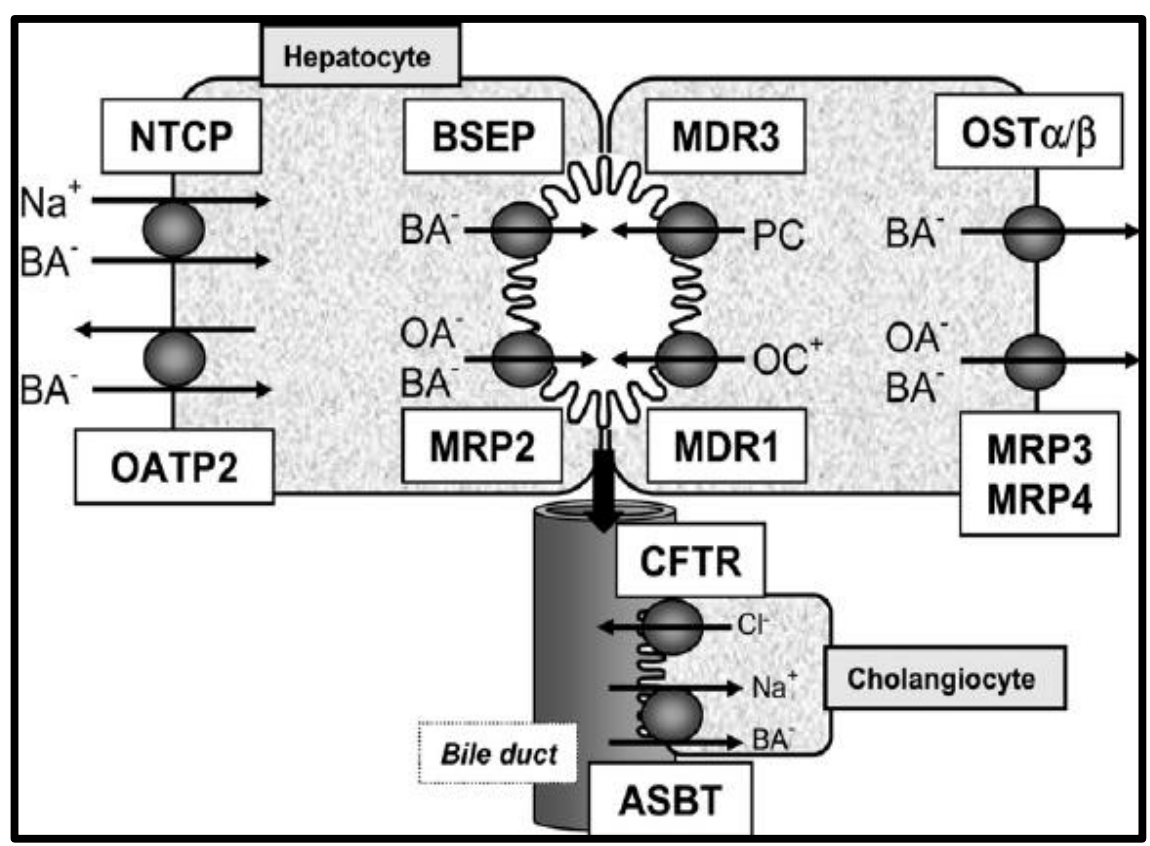

Figure (1): The bile canalicular and the major hepatobiliary transport systems.

Hepatobiliary transport systems in hepatocytes and cholangiocytes. Bile acids are taken up by the NTCP and OATPs at the basolateral membrane of hepatocytes and exported into bile by the BSEP and the MRP2. MRP2 also mediates excretion of various anions (OA), such as bilirubin, into the canaliculus. The phospholipid export pump MDR3 mediates excretion of phosphatidylcholine (PC), which forms mixed micelles together with bile acids and cholesterol in bile. Cationic drugs are excreted by the MDR1. At the basolateral membrane of hepatocytes, MRP3, MRP4, and the heteromeric organic solute transporter $\alpha / \beta$ provide an alternative excretion route for bile 
acids and other OA into the systemic circulation. Quoted from Zollner and Trauner, (2008).

\section{Basolateral (sinusoidal) bile acid transporters.}

The basolateral membrane transporters uptake bile acids from the portal blood into the liver and does not require active transport; electroneutrality is sufficient (Balistreri et al., 2005). Some of these transporters are exchangers that mediate organic anion uptake in exchange for bicarbonate or glutathione. These proteins belong to the superfamily of solute carriers OATPs named OATP-1, OATP-2, OATP-4, and OATP-8 (Stieger, 2011).

The NTCP is another transporter, which carries only bile acids. It cotransports two $\mathrm{Na}^{+}$down its gradient into the hepatocytes along with one molecule of conjugated bile acid (Stieger, 2011). MRP3 cotransports conjugates such as bilirubin monoglucuronide and diglucuronide. Although MRP3 is a member of multidrug resistance associated proteins, it is localized exclusively in the basolateral plasma membrane instead of the apical membrane (Keppler, 2011).

Unconjugated bilirubin, a constituent of the bile, is bound with serum albumin and cannot pass through the kidney. Normally, unconjugated bilirubin is converted in the endoplasmic reticulum of the hepatocytes to monoglucuronosyl bilirubin and bisglucuronosyl bilirubin by uridine diphosphate glucuronosyltransferase (UDPglucuronosyltransferase), followed by MRP2-mediated efflux across the canalicular membrane into the bile. However, when the formation or the uptake of bilirubin glucuronides exceed the transport capacity of MRP2 in the canalicular membrane, the sinusoidal efflux via MRP3 provides a pathway for compensation (Keppler, 2014).

Defect in the expression and/or the function of liver efflux transporters is associated with intrahepatic cholestasis. This was proved by determining that the genetic defects in human BSEP, ATPase phospholipid transporting 8B1 (ATP8B1), or MDR3 (human ortholog of rat MDR2) correlate to progressive familial intrahepatic cholestasis (type I, II, and III, respectively). In addition, a genetic defect in human MRP2 is a main cause of Dubin-Johnson Syndrome, resulting in chronic hyperbilirubinemia and jaundice. The antidiabetic drug, troglitazone, was known to induce intrahepatic cholestasis resulting from BSEP inhibition. An inability to induce MRP2, BSEP, MDR2, and ATP8B1 mRNA expression, as well as a further decrease in BSEP protein expression appears to reduce hepatocyte viability and function (Cui et al., 2009).

\section{Regulation of bile acids.}

Regulation of bile acids homeostasis and their secretion under physiologic and pathologic conditions involve a complex network of nuclear receptors (NRs), which are present in the liver (Wagner et al., 2011). NRs are ligand-modulated transcription factors which are involved in the expression of metabolic enzymes and transporters, thus, regulate several hepatic processes, such as hepatobiliary excretory function, hepatic glucose and lipid metabolism, inflammation, regeneration, fibrosis and tumorigenesis (Trauner and Halilbasic, 2011).

The NRs, which are normally present in the cytoplasm, are transported into the nucleus either directly by binding to ligands or indirectly by signal-mediated interactions. This is followed by heterodimerization between the NRs and the retinoid X 
receptor, which is normally present in the nucleus. These heterodimers bind to specific gene promoters and alter the expression of the target genes by interfering with their transcription (Trauner and Halilbasic, 2011).

For instance, nuclear receptors that are related to the regulation of hepatobiliary homeostasis, bile synthesis, and bile secretion include the Farnesoid X receptor (FXR), Short heterodimer partner (SHP), Pregnane X receptor (PXR), and Vitamin D receptor. As regulators of inflammation, fibrosis, and energy homeostasis, NRs such as Glucocorticoid receptor, peroxisome proliferator-activated receptors alpha (PPAR $\alpha$ ), and peroxisome proliferator-activated receptors gamma (PPAR $\gamma$ ) can also contribute to cholestatic liver disease. Furthermore, other biliary constituents such as bilirubin can also activate NRs such as the Constitutive Androstane Receptor (CAR) (D'Aldebert et al., 2009).

The most pertinent bile acids-activated NR for regulation of hepatobiliary homeostasis and bile secretion is the FXR (Makishima et al., 1999). Furthermore, other NRs such as PPARs in particular PPAR $\alpha$ have a role in the regulation of inflammation, fibrosis, energy homeostasis and may also have influence on biliary homeostasis and cholestatic liver injury (Kota et al., 2005).

\section{Farnesoid X Receptor (FXR).}

The FXR is predominantly expressed in organs involved in bile acids transport or metabolism such as liver, ileum, kidney and adrenal glands. The hydrophobic bile acids such as CDCA is the most efficacious endogenous FXR ligand, whereas hydrophilic bile acids, such as Ursodeoxycholic acid (UDCA) and muricholic acids, do not activate FXR (Chiang, 2009). When hydrophobic bile acids are accumulated in the liver, FXR is activated to encompass the regulation of the enterohepatic circulation and intracellular load of bile acids (Chiang, 2004).

After ligand (bile acids) binding to FXR, it binds to DNA elements called FXR response elements (FXREs). Then, FXR could activate or repress gene transcription through a variety of FXREs either as a monomer or as a FXR/RXR heterodimer. The inhibitory effects of FXR may be also linked to FXR-mediated induction of an atypical NR (SHP). SHP in turn, interacts with 2 other NRs that control CYP7A1 expression, the hepatic nuclear factor 4 (HNF4 or NR2A1) and the liver receptor homolog-1 (LRH-1 or NR5A2) through promoting the dissociation of coactivators linked to them (Goodwin et al., 2000; Kir et al., 2012) (Figure 2). 


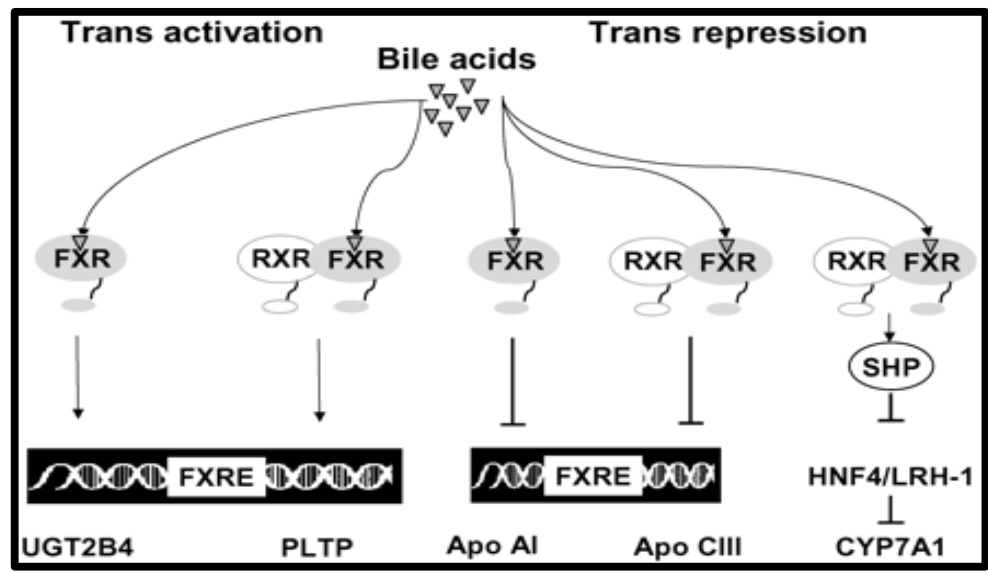

Figure (2): Various modes of FXR gene regulation. FXR can induce transcription as a monomer or a heterodimer with RXR. FXR can also repress transcription indirectly through the induction of SHP, or directly as a monomer or a heterodimer, Quoted from Claudel et al., (2005).

Activation of FXR leads to the inhibition of the basolateral uptake transporter NTCP activity, thus limiting bile acids uptake from the sinusoidal blood to reduce the hepatocellular bile acids level (Denson et al., 2001). In a way of promoting the bile acids biliary excretion, FXR activation upregulates the canalicular export transporter BSEP as well as MRP2 in hepatocytes (Gerloff et al., 2002; Kubitz et al., 2012). In addition, FXR reduces endogenous bile acid synthesis via classical pathway through the inhibition of rate-limiting enzyme CYP7A1, and via alternative pathways through the inhibition of CYP8B1 and CYP27A1 (Eloranta and Kullak-Ublick, 2005) (Figure 3).

Hepatic FXR stimulates bile secretion not only through regulation of bile acids export but also via induction of MDR2. Thus, the regulatory role of FXR in secretion of biliary phospholipids may be critical for the protection of hepatocytes canalicular membrane as well as the apical membrane of bile duct lining cells against the detergent properties of secreted bile acids (Huang et al., 2003).

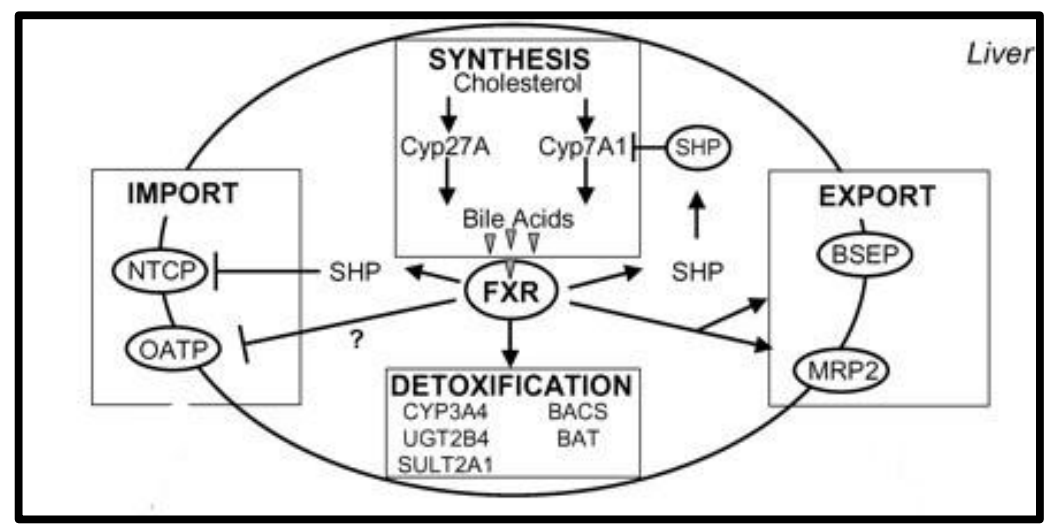

Figure (3): Overview of bile circulation within the hepatocyte mebranes. FXR, negatively regulates bile acid production by repressing CYP7A1 the rate-limiting enzyme of the synthetic pathway. The FXR induces the expression of bile acid-CoA synthetase (BACS) and bile acid-CoA:amino acid $N$-acetyltransferase (BAT), which are 
involved in bile acid conjugation. The FXR activates the expression of the bile acid export transporters (MRP2 and BSEP) and simultaneously represses bile acid import by downregulation of NTCP and possibly organic anion transporter C (OATP-C). Quoted from Claudel et al.,(2005).

Another role of FXR, is conjugation of bile acids to taurine or glycine by sequential actions of the enzymes: the BACS and the BAT, to increase their hydrophilicity and consequently decrease their toxicity (Pircher et al., 2003) (Figure 3).

\section{Cholestatic Liver injury.}

Cholestasis is characterized by bile flow stagnation that can be the result of a failure in synthesis of bile acids, a dysfunction in the transporters of hepatocytes or ductular cells or from a physical blockade of the external bile ducts. The former is considered intrahepatic and the latter is extrahepatic cholestasis (Qureshi, 1999).

Interaction with bile acid transporters is a mechanism that has been observed under administration of several drugs including cyclosporine A, rifampicin, glibenclamide, chlorpromazine, erythromycin and oxypenicillins leading to intrahepatic cholestasis (Rodriguez-Garay, 2003). Estrogens are a known cause of intrahepatic cholestasis during pregnancy in susceptible women or during administration of oral contraceptives and post-menopausal hormone replacement therapy. Intrahepatic cholestasis is characteristic as well in some congenital disorders such as idiopathic neonatal hepatitis or during the course of viral hepatitis (Qureshi, 1999).

However, cholestasis may develop from mechanical obstructions in the extrahepatic bile ducts which is characteristic to certain autoimmune diseases such as in primary biliary cholangitis (PBC), primary sclerosing cholangitis (PSC) and biliary atresia (specific to newborns). A stone or a tumour at the level of the extrahepatic bile ducts usually leads to extrahepatic cholestasis (Poupon et al., 2000).

Eventually, cholestasis results in a dramatic increase in liver and serum bile acids levels which is potentially toxic to living cells. The mechanism by which bile acids induce toxicity could be possibly due to the detergent nature of bile acids, which seems to solubilize plasma membranes and leads to cell death which in turn progresses to oxidative stress, oxidation of reduced glutathione, and lipid peroxidation (Baron and Muriel, 1999). Without proper treatment, cholestasis will be followed by jaundice and hypercholesterolemia, and then aggravated outcomes as development of fibrosis and cirrhosis (Boyer, 2007).

Jaundice occurs due to the accumulation of bilirubin as a result of failure of damaged liver cells to convert bilirubin into bilirubin glucuronides to be transported into the bile canaliculi (Chaudhuri, 2006).

The common complications associated with cholestasis include pruritus, osteoporosis, dyslipidemia and vitamin deficiencies (Ahmed and Mayo, 2014).

\section{Experimental models of Cholestasis.}

The most common experimental models of intrahepatic cholestasis are estrogeninduced, endotoxin-induced and drug-induced cholestasis (e.g., erythromycin, chlorpromazine, anti-tuberculosis drugs and others). A well-known model of extrahepatic cholestasis is common bile duct ligation (BDL) (Rodriguez-Garay, 2003). 
Alpha-naphthylisothiocyanate (ANIT)-induced intrahepatic cholestasis is extensively used in experimental animals, which is useful for studying the mechanisms of drug-induced cholestasis, because cholestasis and hepatic damage resulting from the administration of certain drugs to humans are mimicked by ANIT injection to rats (Kossor et al., 1993).

Liver injury and pathological changes induced by increased hepatocellular bile acids.

\section{Oxidative stress.}

Oxidative stress likely plays a large role in cholestatic liver injury, possibly as a result of mitochondrial membrane damage associated with necrosis. In hepatocytes, high concentrations of bile acids can cause damage to the basolateral membrane and cell organelle membranes, also because they are more concentrated in the bile, they are more harmful to the canalicular membrane. The effect of bile acids on cell membranes is induced by binding to the membrane lipids and forming aggregates. When bile acids concentration increases, it results in the formation of membrane holes, which cause disruption of cell membrane integrity (Schubert and Schmidt, 1988).

One of the important organelles is the mitochondria, where disruption of its membrane by bile acids leads to impaired respiration and electron transport (Hofmann, 1999). Hydrophobic bile acids decrease the activities of several enzyme complexes involved in the electron transport chain, such as complexes I, III and IV, a process that can increase generation of reactive oxygen species (ROS) by mitochondria (Krahenbuhl et al., 1994). The result of oxygen radical formation is damage to a variety of biomolecules found in tissues, including nucleic acids, membrane lipids (lipid peroxidation), enzymes and receptors leading to further disruption of membrane fluidity and enhancement of cell lysis (Stark, 2005).

The resultant oxidative stress also lead to depletion of mitochondrial antioxidants, mainly glutathione and superoxide dismutase (SOD), or directly oxidize thiol groups in the mitochondrial permeability transition pore protein (MPTP), leading to mitochondrial permeability transition (MPT) (Costantini et al., 1996). The MPT is a rapid increase in the permeability of the inner mitochondrial membrane to solutes of low molecular mass, which results in collapsing of the electrochemical gradient across the inner membrane, uncoupling of oxidative phosphorylation, and colloid-osmotic swelling of mitochondria (Zoratti and Szabo, 1995; Bernardi and Forte, 2007) . Induction of the MPT precedes the onset of cell necrosis (Lemasters et al., 2009) or apoptosis (Susin et al., 1998).

The MPT may lead to cellular necrosis through NADP $(\mathrm{H})$ oxidation, reducing capacity for oxidative phosphorylation and depletion of cellular ATP, alterations in cellular calcium homeostasis, and plasma membrane structural changes (Lemasters et al., 2009). Apoptosis may involve mitochondrial proteases (Gores et al., 1998), protein kinase C (Jones et al., 1997), the Fas signaling pathway, downstream caspases (Faubion et al., 1999) or translocation of Bax to mitochondria (Rodrigues et al., 1998).

The determinant of whether necrosis or apoptosis will occur after induction of the MPT may be the residual cellular ATP levels. If a large number of mitochondria in a given cell undergo the permeability transition and cellular ATP levels become depleted 
(common in acute cholestasis), necrosis is favoured, whereas maintenance of ATP levels when a fewer number of mitochondria undergo the MPT, the cell will favour apoptosis (common in chronic cholestasis) (Lemasters et al., 1998).

Any tissue trauma or excessive stress with cell injury will trigger an acute inflammatory response.

\section{Inflammation and neutrophil infiltration.}

The main purpose of inflammation is the activation of the innate immune response to eliminate invading microorganisms and foreign objects, to remove damaged cells and cell debris and to prepare the tissue for regeneration. Virtually, excessive inflammatory response causes additional liver damage or even triggers liver failure (Jaeschke et al., 2002).

Necrotic cells release inflammatory mediators such as high mobility group box 1 protein (HMGB1) (Scaffidi et al., 2010), in addition to other products of damaged cells, i.e. lipid peroxidation products, and ROS (Curzio et al., 1986) which can trigger more classical inflammatory mediators through a toll like receptor (TLR4) mediated pathway that is highly expressed in Kupffer cells (Makled et al., 2016) (Figure 4).

Kupffer cells, the resident macrophages in the liver, are critical for the rapid clearance of microorganisms from the systemic circulation (Gregory and Wing, 2002). Although Kupffer cells by themselves are highly phagocytic and are able to remove microorganisms, they also facilitate generation of inflammatory mediators including tumor necrosis factor $\alpha$ (TNF- $\alpha$ ), interleukins (IL-1, and IL-6), chemokines and reactive oxygen species (ROS). These mediators lead to recruitment of inflammatory cells such as neutrophils, monocytes, $\mathrm{T}$ and B lymphocytes, as well as natural killer cells to the liver (Nagy, 2015).

The TNF- $\alpha$, which is a major mediator of systemic inflammation leads to activation of nuclear factor kappa-B $(\mathrm{NF}-\kappa \mathrm{B})$ in the hepatocytes through phosphorylation of its inhibitory protein kappa B-alpha $(\mathrm{I} \kappa \mathrm{B}-\alpha)$ by inhibitory kappa $\mathrm{B}$ kinase (IKK). Sequentially, NF- $\kappa B$ translocates from the cytoplasm to the nucleus, where it attaches to $\kappa \mathrm{B}$ binding sites and triggers the transcription of more proinflammatory cytokines such as TNF- $\alpha$, IL-1 and chemokines (Li et al., 2013), adhesion molecules and apoptotic factors (Liu et al., 2015).

Inflammatory mediators can promote neutrophil accumulation within the hepatic microvasculature (sinusoids and postsinusoidal venules) (Bajt et al., 2001). These mediators up-regulate the expression of Mac-1 (CD11b/CD18), a member of the $\beta 2$ integrin family of adhesion molecules on neutrophils, resulting in priming and activation of these phagocytes. However, neutrophil activation/accumulation within sinusoids and postsinusoidal venules typically does not cause liver damage. Extravasation into the parenchyma is necessary for neutrophils to cause tissue damage. This step could be initiated by chemotactic factors including CXC chemokines, TNF- $\alpha$, lipid peroxidation products or HMGB1 (Jaeschke and Hasegawa, 2006).

Once neutrophils receive a signal, they adhere to distressed hepatocytes through their $\beta 2$ integrins and intracellular adhesion molecules 1 (ICAM-1) expressed on hepatocytes endothelial cells. The firm adhesion and transmigration processes trigger the exocytosis of gelatinase granules from neutrophils, which liberates matrix metalloproteinases. These enzymes may help in degrading the extracellular matrix 
proteins and facilitate the migration into the parenchyma (Faurschou and Borregaard, 2003).

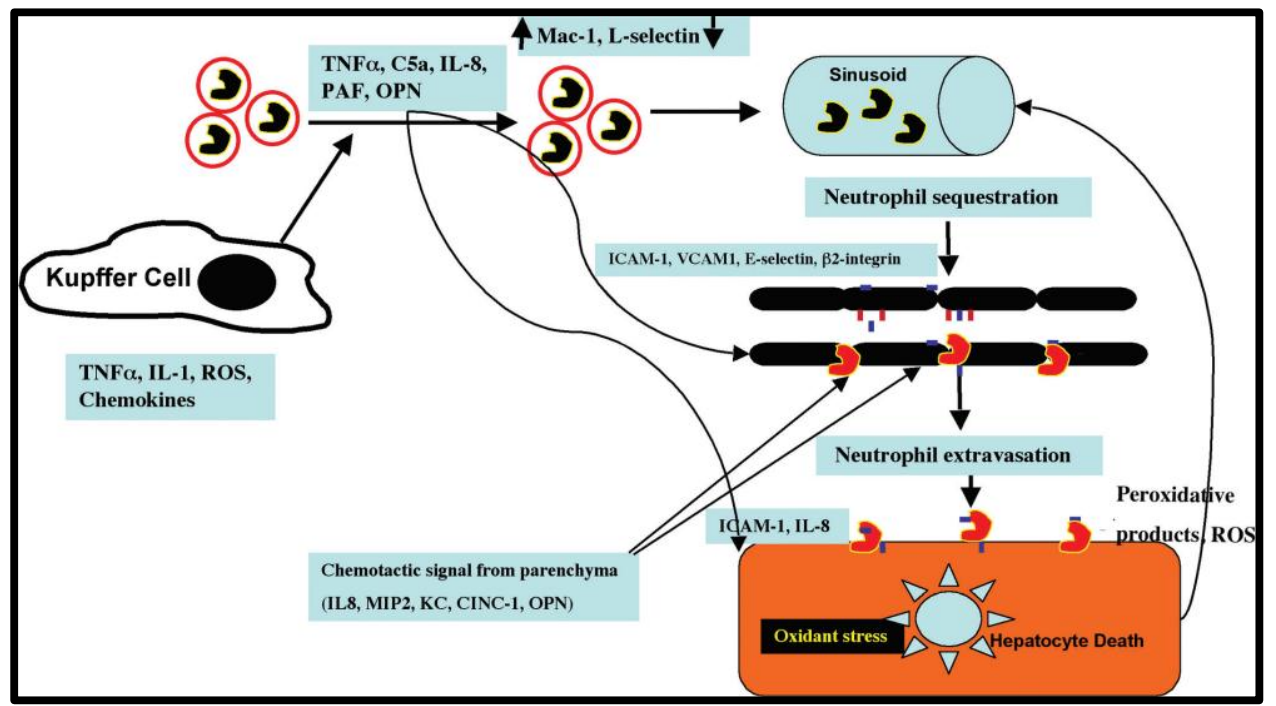

Figure (4): Induction of neutrophil extravasation and the mechanism for neutrophil-mediated liver pathology. Quoted from Ramaiah and Jaeschke,(2007).

Abbreviations: cytokine-induced neutrophil chemoattractant-1 (CINC-1); intracellular adhesion molecules 1 (ICAM-1); vascular cell adhesion molecule 1 (VCAM-1); interleukin 1 (IL-1); interleukin 8 (IL-8); keratinocyte-derived chemokine (KC); Mac-1; macrophage inflammatory protein-2 (MIP2); osteopontin (OPN); platelet activating factor (PAF); reactive oxygen species (ROS); tumor necrosis alpha (TNF- $\alpha$ ).

\section{Neutrophil-induced cell injury.}

The ROS play a role in the recruitment of neutrophils into damaged tissue, but activated neutrophils are also a potential source of ROS (Jaeschke and Hasegawa, 2006).

Neutrophils generate superoxide through NADPH oxidase, which is a multicomponent enzyme system that assembles at the mitochondrial membrane. The SOD spontaneously converts superoxide to oxygen and hydrogen peroxide, which is a highly diffusible oxidant. It is further used by myeloperoxidase, released from the primary granules of neutrophils, to generate hypochlorous acid $(\mathrm{HOCl})$, which is a potent oxidant and chlorinating agent (El-Benna et al., 2005). It also can react with amino groups and form toxic chloramines (Woolbright and Jaeschke, 2012).

Proteases released from neutrophils are clearly involved in cell killing during a neutrophilic hepatitis and also have a role in continuous stimulation of inflammatory cytokines and chemokines (Faurschou and Borregaard, 2003). The neutrophilmediated oxidant stress induces further mitochondrial dysfunction in hepatocytes. This can eventually triggers the opening of the MPTP and the collapse of the MPT resulting in oncotic necrosis (Bernardi and Forte, 2007) (Figure 5). 
Additionally, TNF- $\alpha$ and IL-1beta downregulate OATP2 and MRP2, which are responsible for transport of bile acids, bilirubin and other organic ions. Thus, elevated bile acids concentrations during cholestasis might cause a secondary downregulation of bile acid transporters by mediation of inflammatory cytokines (Hartmann et al., 2002).

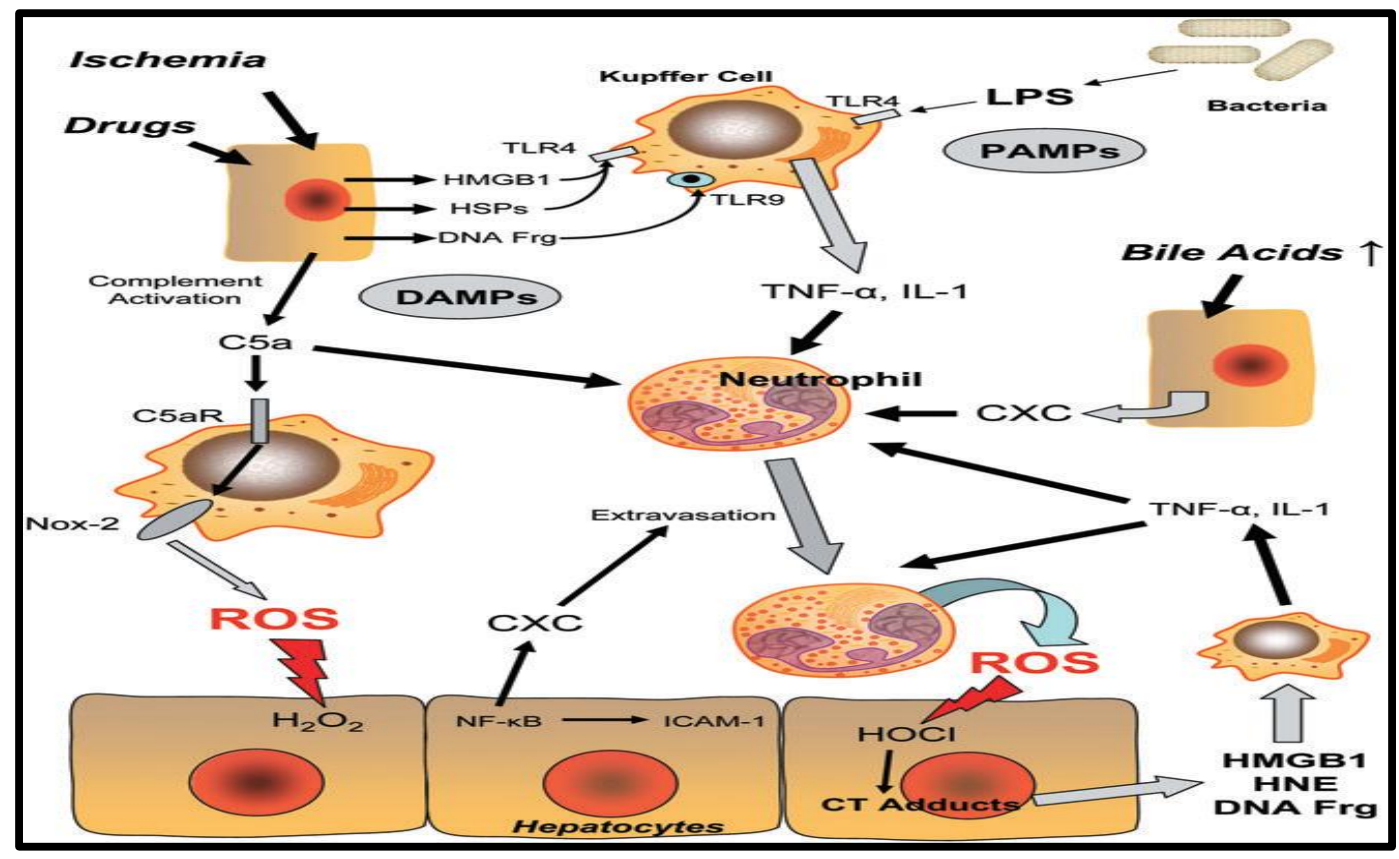

Figure (5): Mechanisms of an acute inflammatory response after cellular stress as in drug toxicity and cholestasis. Quoted from Jaeschke,(2011).

Abbreviations: CXC, CXC chemokines (IL-8); C5aR, complement fragment 5a receptor; CT adducts, chlorotyrosine protein adducts; DAMPs, damage-associated molecular patterns; DNA Frg, DNA fragments; HMGB1, high mobility group box 1 protein; HNE, hydroxynonenal (lipid peroxidation product); HSPs. Heat shock proteins; $\mathrm{HOCl}$, hypochlorous acid; ICAM-1, intercellular adhesion molecule-1; IL-1, interleukin-1; LPS, lipopolysaccharide/endotoxin; NF-kB, nuclear factor kB; Nox-2, NADPH oxidase; PAMPs, pathogenassociated molecular patterns; ROS, reactive oxygen species; TLR, toll like receptor; TNF- $\alpha$, tumor necrosis factor- $\alpha$.

\section{Management of cholestatic liver injury.}

There are many methods to reduce cholestasis; prevention of the oxidative stress through antioxidants, acceleration of the metabolism of bilirubin, enhancement of the excretion of bile acids and reduction of the inflammation. In clinical practice, ursodeoxycholic acid (UDCA) is the representative drug frequently used to treat cholestatic liver injury (Cies and Giamalis, 2007). UDCA is the 7- $\beta$ epimer of the primary human bile acid CDCA, is a hydrophilic bile acid that is mainly absorbed in the small intestine, transported into the liver through the portal circulation (with a 50\% first pass extraction rate), conjugated with glycine and taurine, and actively secreted into bile. Conjugated UDCA competes with endogenous bile acids for active transport into portal bloodstream and undergoes enterohepatic recirculation, whereas non-absorbed 
UDCA molecules are de-conjugated, then converted into LCA by intestinal bacteria and finally eliminated into stools. Usually UDCA is administered at the recommended adult dosage of 13-15 mg/kg/day in 2 divided doses (Carey et al., 2015).

Different mechanisms of action are involved in UDCA's beneficial effect a) choleretic and anti-cholestatic effects, due to intracellular molecular signalling pathways that stimulate cellular secretions by promoting vesicular exocytosis and trans-membrane carriers' insertion (Beuers et al., 2015); b) cytoprotection against toxic effects of bile acids and cytokine-induced injury, by stabilization of cell membranes, enhancement of the defences against oxidative stress and inhibition of apoptosis (Beuers, 2006); in addition to this, UDCA contributes to the biliary bicarbonate (HCO3-) umbrella enhancing biliary $\mathrm{HCO} 3-$ secretion against the acidification of the apical surface of cholangiocytes and hepatocytes due to acid BAs (Beuers et al., 2010); c) immunomodulation and anti-inflammatory effects, by inhibiting prostaglandin E2 thus blocking the propagation of autoimmune liver injury; it also decreases eosinophil levels in blood stream, and suppresses the immune reaction against PAMPs such LPS's Lipid A; d) increase of the hydrophilicity of the circulating endogenous bile acids pool as well as up-regulating liver glutathione synthesis (Poupon, 2012).

However, UDCA treatment needs tedious course and some patients do not respond to treatment (especially patients of PBC) (O'Leary and Pratt, 2007). Hence, there are several directions to find drugs with low side effects and prompt effect for treating cholestatic liver injury.

Understanding NR function not only increases our understanding of the physiology and pathophysiology of bile acids metabolism, but also can lead to development of NR ligands for the treatment of cholestasis. Interestingly, many reports demonstrate alterations in transcriptional coactivators of FXR in cholestatic liver diseases. For instance, Peroxisome proliferator-activated receptor gamma coactivator 1alpha (PGC1- $\alpha$ ) expression is repressed in patients with gallstones (Xu et al., 2012). This suggests that PGC1- $\alpha$ associated reduction of FXR activity could contribute to altered bile composition and gallstone formation through inhibition of target genes BSEP and MDR3 (Cho and Boyer, 1999). Thus, pharmacological stimulation of BSEP or FXR presents as a potential therapeutic option for treating cholestatic liver diseases. FXR also represses transcription of CYP7A1 (an enzyme that mediates rate-limiting step in conversion of cholesterol to bile acids) through SHP (Gadaleta et al., 2011). FXR induces fibroblast growth factor (Fgf-15) in the small intestine and represses CYP7A1 in liver through a mechanism that involves FGF receptor 4 (FGFR4) and SHP (Modica et al., 2012). In addition to FXR, genetic variants of PXR are also associated with increased susceptibility to cholestatic liver disease such as intrahepatic cholestasis of pregnancy and PSC. An increased expression in PXR and CAR is also evidenced in patients with obstructive cholestasis, and the expression decreases in late-stage cholestasis for limiting the progression of liver injury (Fiorucci et al., 2004).

Actually, the main investigational targets for treatment of cholestasis and PBC are: i) the farnesoid $\mathrm{X}$ receptor (FXR); ii) the Pregnane $\mathrm{X}$ Receptor (PXR); iii) the Constitutive Androstane Receptor (CAR); iv) the peroxisome proliferator activated receptor (PPAR). 


\section{Farnesoid X Receptor}

One of the main NRs that regulate bile acid formation, transportation and metabolism is the FXR. Thus it should be a target for treating cholestatic diseases. Activation of FXR could efficiently prevent cholestasis through several mechanisms:

1. Inhibition of several enzymes involved in the synthesis of bile acid from cholesterol (mainly CYP7A1).

2. increase of bile acids export (through upregulation of BSEP on the canalicular membrane).

3. Producing a less toxic bile by changing bile composition (through increasing relative phospholipid and bicarbonate secretion to maintain normal micellar concentration).

4. Mediating direct anti-inflammatory effects in hepatocytes (via inhibition of NF$\kappa \mathrm{B})$ and non-parenchymal liver cells (Halilbasic et al., 2013).

A recent potent and selective agonist of FXR was detected known as 6a-ethylchenodeoxycholic acid or obeticholic acid (OCA). It is a modified bile acid and FXR agonist that is derived from primary human bile acid chenodeoxycholic acid (being an endogenous ligand of FXR). OCA was effectively able to alleviate Alanine aminotransferase (ALT) levels as well as pruritis in PBC which is a challenging cholestatic disease. (Kowdley et al., 2018).

Recently, a phase 3, double-blind, placebo-controlled trial and long term extension OCA in patients with PBC has been published (Kowdley et al., 2018). Two hundred and seventeen patients with inadequate response to UDCA or intolerant to UDCA were randomly assigned to receive OCA at a dose of $10 \mathrm{mg}$, or to an OCA titration group (starting with $5 \mathrm{mg}$ with adjustment to $10 \mathrm{mg}$ ) or placebo (indeed this group continued to receive UDCA). The primary end-point was an alkaline phosphatase (ALP) level of b1.67 times the upper limit of the normal range, with a reduction of at least $15 \%$ from baseline, and a normal total bilirubin level. The best predictive parameter of response was the significant reduction in ALP within 2 weeks of treatment and at every point thereafter. Pruritus was the major side-effect occurring in the groups treated with OCA, but only one patient dropped out the study for this symptom.

Indeed, pruritus, slowly decreased within the months of treatment, and at the end of 12 months was acceptable and similar in all the three groups of treatment.

OCA has been approved in May 2016 by food and drug administration for patients not responding or intolerant to UDCA and the European Commission granted a marketing authorization valid throughout the European Union for OCA in December 2016 (Floreani and Mangini, 2018).

\section{Pregnane X Receptor}

The PXR agonists such as atorvastatin and pregnenolone were investigated for their effect on metabolism of bile acids and bilirubin as well as stimulation of hepatic efflux system. The studies demonstrated that atorvastatin did not cholestasis in PBC patients (Halilbasic et al., 2013).

\section{Constitutive Androstane Receptor}

6-(4-Chlorophenyl)imidazo[2,1-b][1,3]thiazole-5-carbaldehyde-O-(3,4dichlorobenzyl) oxime (CITCO), a CAR activator, also protects against cholestasis (Huang et al., 2003). In addition, CAR agonists phenobarbital and 1,4-bis-[2-(3,5- 
dichlorpyridyloxy)]benzene reduced serum bilirubin and bile acids levels in healthy as well as in BDL mice. Although promising, the efficaciousness of CAR agonists in the treatment of cholestatic liver disease calls for more research (Wada et al., 2009).

\section{Peroxisome proliferator activated receptor (PPAR)}

An interesting class of agents is represented by fibrates which induce PPARaUDP glucuronyl- transferase (UGTS) signalling axis which is important for several actions: a) reducing inflammation and autoantibody production by inhibiting NF-kB translocation with consequent VCAM-1 expression that decreases leukocyte adhesion and transendothelial migration; b) promoting the expression of MDR3 and subsequently inducing biliary phospholipid secretion; c) down-regulating of different transporters (SULT 2A1, ASBT, MRP3, MRP4); d) altering the ratio of CA / CDCA by promoting the expression and activity of CYP8B1(Ghonem et al., 2015).

A number of small, clinical, observational studies have assessed the potential efficacy of fibrates in PBC, the majority coming from Japan. A meta-analysis including 6 randomized studies with a total number of 84 patients concluded that combination therapy with UDCA plus fenofibrate is more effective that UDCA alone in reducing ALP, Gamma glutamyl transferase, immunoglobulin $M$ and triglycerides but not pruritus in PBC (Zhang et al., 2015).

A more recent retrospective paper performed at a tertiary centre in Toronto compared outcomes between a group of patients treated with fenofibrate and UDCA or UDCA alone (Cheung et al., 2016). A biochemical response evaluated according to the Toronto criteria was reached by $41 \%$ vs $7 \%$ respectively. Fenofibrate was also associated with improved decompensation-free and transplant-free survival. Moreover, only fenofibrate use, not biochemical response, was independently associated with improved outcomes on multivariate analysis. However, an editorial on the use of fenofibrate as second-line therapy in high risk PBC, raised some caution thereafter (Jones and Hansen, 2016), considering that in the Canadian study bilirubin increased more rapidly in cirrhotic patients treated with the combined therapy UDCA + fenofibrate than in the group treated with UDCA alone (Zhang et al., 2015).

Another interesting retrospective study has been performed in the multicentre cohort of the Global PBC Study Group (Zhao et al., 2017). Two hundred and eleven patients were treated with fibrates plus UDCA (138 with fenofibrate and 73 with bezafibrate) with a median follow-up after fibrate initiation of 31 months. After 12 months, a significant reduction compared to baseline of ALP (p 0.001), ALT ( $p$ 0.001), and Aspartate aminotransferase $(p=b$ 0.002) were observed. Bilirubin did not significantly change and 5-year survival was $91 \%$ vs $84 \%$.

Finally, the first large, randomized placebo-controlled trial of fibrate therapy in patients with $\mathrm{PBC}$ has been presented at "European association for the study of the liver" in Amsterdam (Corpechot et al., 2017). Hundred patients with inadequate biochemical response to UDCA have been randomized in two arms: a) placebo and b) $400 \mathrm{mg}$ bezafibrate. A premature termination was recorded in 6 and in 2 patients respectively. At baseline the two groups were homogeneously balanced in terms of age, female prevalence, liver function tests, liver stiffness and prevalence of advanced stage (52\% and 56\% respectively).

After 2 years a complete biochemical response was reached in $30 \%$ in the group treated with bezafibrate and in none of the patients in the group with placebo ( $\mathrm{p}$ 0.0001). 
Normalization of ALP was reached in $67 \%$ in the group with bezafibrate vs $0 \%$ in the group with placebo. Improvement in pruritus was achieved more frequently in the bezafibrate than in the placebo group (Corpechot et al., 2017).

\section{REFERENCES}

Ahmed, A.; Mayo, M. (2014) Complications of Cholestasis. In: Cholestatic Liver Injury, $2^{\text {nd }}$ Edn.; Carey, E.; Lindor, K., Eds.; Springer, New York, USA, 2014, pp. 163-182.

Alrefai, W.; Gill, R. (2007) Bile acid transporters: structure, function, regulation and pathophysiological implications. Pharm. Res., 24, (10), 1803-1823.

Bajt, M.; Farhood, A.; Jaeschke, H.(2001) Effect of CXC chemokines on neutrophil activation and sequestration in hepatic vasculature. Am. J. Physiol. Gastrointest. Liver. Physiol., 281, (5), 1188-1195.

Balistreri, W.; Bezerra, J.; Jansen, P.; Karpen, S.; Shneider, B.; Suchy, F.(2005) Intrahepatic cholestasis: summary of an American Association for the Study of Liver Diseases single-topic conference. Hepatology., 42, (1), 222-235.

Baron, V.; Muriel, P. (1999) Role of glutathione, lipid peroxidation and antioxidants on acute bile-duct obstruction in the rat. Biochim. Biophys. Acta., 1472, (1-2), 173-180.

Bernardi, P.; Forte, M.(2007) The mitochondrial permeability transition pore. Novartis. Found. Symp., 287, 157-164.

Beuers, U.(2006) Drug insight: Mechanisms and sites of action of ursodeoxycholic acid in cholestasis. Nat Clin Pract Gastroenterol Hepatol., 3, (6), 318-328.

Beuers, U.; Hohenester, S.; de Buy Wenniger, L.; Kremer, A.; Jansen, P.; Elferink, R.(2010) The biliary $\mathrm{HCO}(3)(-)$ umbrella: a unifying hypothesis on pathogenetic and therapeutic aspects of fibrosing cholangiopathies. Hepatology., 52, (4), 1489-1496.

Beuers, U.; Trauner, M.; Jansen, P.; Poupon, R. (2015) New paradigms in the treatment of hepatic cholestasis: from UDCA to FXR, PXR and beyond. $J$ Hepatol., 62, (1 Suppl), S25-37.

Boyer, J.(1980) New concepts of mechanisms of hepatocyte bile formation. Physiol. Rev., 60, (2), 303-326.

Boyer, J. (2007) New perspectives for the treatment of cholestasis: lessons from basic science applied clinically. J. Hepatol., 46, (3), 365-371.

Carey, E.; Ali, A.; Lindor, K.(2015) Primary biliary cirrhosis. Lancet., 386, (10003), 1565-1575.

Chaudhuri, S., (2006) Ed. The Liver. In: Concise Medical Physiology, $7^{\text {th }}$ Edn.; New Central Book Agency (P) Limited, London, , pp. 105- 112.

Cheung, A.; Lapointe-Shaw, L.; Kowgier, M.; Meza-Cardona, J.; Hirschfield, G.; Janssen, H.; Feld, J. (2016) Combined ursodeoxycholic acid (UDCA) and fenofibrate in primary biliary cholangitis patients with incomplete UDCA response may improve outcomes. Aliment Pharmacol Ther., 43, (2), 283-293. 
Chiang, J. (2002) Bile acid regulation of gene expression: roles of nuclear hormone receptors. Endocr. Rev. 2002, 23, (4), 443-463.

Chiang, J. (2009) Bile acids: regulation of synthesis. J. Lipid. Res., 50, (10), 19551966.

Chiang, J. (2004) Regulation of bile acid synthesis: pathways, nuclear receptors, and mechanisms. J. Hepatol., 40, (3), 539-551.

Cho, W.; Boyer, J. (1999) Vasoactive intestinal polypeptide is a potent regulator of bile secretion from rat cholangiocytes. Gastroenterology., 117, (2), 420-428.

Cies, J.; Giamalis, J. (2007) Treatment of cholestatic pruritus in children. Am. J. Health. Syst. Pharm., 64, (11), 1157-1162.

Claudel, T.; Staels, B.; Kuipers, F. (2005) The Farnesoid X receptor: a molecular link between bile acid and lipid and glucose metabolism. Arterioscler. Thromb. Vasc. Biol., 25, (10), 2020-2030.

Corpechot, C.; Chazouillères, O.; Rousseau, A.; Guyader, D.; Habersetzer, F.; Mathurin, P.; Goria, O.; Potier, P.; Minello, A.; Silvain, C.; Abergel, A.; Debette-Gratien, M.; Larrey, D.; Roux, O.; Bronowicki, J.; Boursier, J.; de Ledhingen, V.; Heurgue-Berlot, A.; Nguyen-Khac, E.; Zoulim, F.; Ollivier-Hourmand, I.; Zarski, J. P.; Nkontchou, G.; Gaouar, F.; Simon, T.; Poupon, R. (2017) A 2-year multicenter, double-blind, randomized, placebo-controlled study of bezafibrate for the treatment of primary biliary cholangitis in patients with inadequate biochemical response to ursodeoxycholic acid therapy (Bezurso). Journal of Hepatology., 66, (1), S89.

Costantini, P.; Chernyak, B.; Petronilli, V.; Bernardi, P. (1996) Modulation of the mitochondrial permeability transition pore by pyridine nucleotides and dithiol oxidation at two separate sites. J. Biol. Chem., 271, (12), 6746-6751.

Cui, Y.; Aleksunes, L.; Tanaka, Y.; Goedken, M.; Klaassen, C. (2009) Compensatory induction of liver efflux transporters in response to ANITinduced liver injury is impaired in FXR-null mice. Toxicol. Sci., 110, (1), 4760 .

Curzio, M.; Esterbauer, H.; Di Mauro, C.; Cecchini, G.; Dianzani, M. (1986) Chemotactic activity of the lipid peroxidation product 4-hydroxynonenal and homologous hydroxyalkenals. Biol. Chem. Hoppe. Seyler., 367, (4), 321-329.

D'Aldebert, E.; Biyeyeme Bi Mve, M.; Mergey, M.; Wendum, D.; Firrincieli, D.; Coilly, A.; Fouassier, L.; Corpechot, C.; Poupon, R.; Housset, C.; Chignard, N. (2009) Bile salts control the antimicrobial peptide cathelicidin through nuclear receptors in the human biliary epithelium. Gastroenterology., 136, (4), 1435-1443.

Denson, L.; Sturm, E.; Echevarria, W.; Zimmerman, T.; Makishima, M.; Mangelsdorf, D.; Karpen, S. (2001) The orphan nuclear receptor, shp, mediates bile acid-induced inhibition of the rat bile acid transporter, ntcp. Gastroenterology., 121, (1), 140-147. 
El-Benna, J.; Dang, P.; Gougerot-Pocidalo, M.; Elbim, C. (2005) Phagocyte NADPH oxidase: a multicomponent enzyme essential for host defenses. Arch. Immunol. Ther. Exp (Warsz)., 53, (3), 199-206.

Eloranta, J.; Kullak-Ublick, G. (2005) Coordinate transcriptional regulation of bile acid homeostasis and drug metabolism. Arch. Biochem. Biophys., 433, (2), 397-412.

Faubion, W.; Guicciardi, M.; Miyoshi, H.; Bronk, S.; Roberts, P.; Svingen, P.; Kaufmann, S.; Gores, G. (1999) Toxic bile salts induce rodent hepatocyte apoptosis via direct activation of Fas. J. Clin. Invest., 103, (1), 137-145.

Faurschou, M.; Borregaard, N. (2003) Neutrophil granules and secretory vesicles in inflammation. Microbes. Infect., 5, (14), 1317-1327.

Fiorucci, S.; Antonelli, E.; Rizzo, G.; Renga, B.; Mencarelli, A.; Riccardi, L.; Orlandi, S.; Pellicciari, R.; Morelli, A. (2004) The nuclear receptor SHP mediates inhibition of hepatic stellate cells by FXR and protects against liver fibrosis. Gastroenterology., 127, (5), 1497-1512.

Floreani, A.; Mangini, C. (2018). Primary biliary cholangitis: Old and novel therapy. Eur J Intern Med. 2018, 47, 1-5.

Gadaleta, R.; van Erpecum, K.; Oldenburg, B.; Willemsen, E.; Renooij, W.; Murzilli, S.; Klomp, L.; Siersema, P.; Schipper, M.; Danese, S.; Penna, G.; Laverny, G.; Adorini, L.; Moschetta, A.; van Mil, S. (2011) Farnesoid X receptor activation inhibits inflammation and preserves the intestinal barrier in inflammatory bowel disease. Gut., 60, (4), 463-472.

Gerloff, T.; Geier, A.; Roots, I.; Meier, P.; Gartung, C. (2002) Functional analysis of the rat bile salt export pump gene promoter. Eur. J. Biochem., 269, (14), 34953503.

Ghonem, N.; Assis, D.; Boyer, J. (2015) Fibrates and cholestasis. Hepatology., 62, (2), 635-643.

Gonzales, E.; Cresteil, D.; Baussan, C.; Dabadie, A.; Gerhardt, M.; Jacquemin, E. (2004) SRD5B1 (AKR1D1) gene analysis in delta(4)-3-oxosteroid 5betareductase deficiency: evidence for primary genetic defect. J. Hepatol., 40, (4), 716-718.

Goodwin, B.; Jones, S.; Price, R.; Watson, M.; McKee, D.; Moore, L.; Galardi, C.; Wilson, J.; Lewis, M.; Roth, M.; Maloney, P.; Willson, T.; Kliewer, S. (2000) A regulatory cascade of the nuclear receptors FXR, SHP-1, and LRH-1 represses bile acid biosynthesis. Mol. Cell., 6, (3), 517-526.

Gores, G.; Miyoshi, H.; Botla, R.; Aguilar, H.; Bronk, S. (1998) Induction of the mitochondrial permeability transition as a mechanism of liver injury during cholestasis: a potential role for mitochondrial proteases. Biochim. Biophys. Acta., 1366, (1-2), 167-175.

Gregory, S.; Wing, E. (2002) Neutrophil-Kupffer cell interaction: a critical component of host defenses to systemic bacterial infections. J. Leukoc. Biol., 72, (2), 239248. 
Halilbasic, E.; Baghdasaryan, A.; Trauner, M. (2013) Nuclear receptors as drug targets in cholestatic liver diseases. Clin. Liver. Dis., 17, (2), 161-189.

Hartmann, G.; Cheung, A.; Piquette-Miller, M. (2002) Inflammatory cytokines, but not bile acids, regulate expression of murine hepatic anion transporters in endotoxemia. J. Pharmacol. Exp. Ther., 303, (1), 273-281.

Hofmann, A. (1999) The continuing importance of bile acids in liver and intestinal disease. Arch. Intern. Med., 159, (22), 2647-2658.

Huang, L.; Zhao, A.; Lew, J.; Zhang, T.; Hrywna, Y.; Thompson, J.; de Pedro, N.; Royo, I.; Blevins, R.; Pelaez, F.; Wright, S.; Cui, J. (2003) Farnesoid X receptor activates transcription of the phospholipid pump MDR3. J. Biol. Chem., 278, (51), 51085-51090.

Huang, W.; Zhang, J.; Chua, S.; Qatanani, M.; Han, Y.; Granata, R.; Moore, D. (2003) Induction of bilirubin clearance by the constitutive androstane receptor (CAR). Proc Natl Acad Sci U S A., 100, (7), 4156-4161.

Jaeschke, H. (2011) Reactive oxygen and mechanisms of inflammatory liver injury: Present concepts. J. Gastroenterol. Hepatol., 26 Suppl 1, 173-179.

Jaeschke, H.; Gores, G.; Cederbaum, A.; Hinson, J.; Pessayre, D.; Lemasters, J. (2002) Mechanisms of hepatotoxicity. Toxicol. Sci., 65, (2), 166-176.

Jaeschke, H.; Hasegawa, T. (2006) Role of neutrophils in acute inflammatory liver injury. Liver. Int., 26, (8), 912-919.

Jones, B.; Rao, Y.; Stravitz, R.; Gores, G. (1997) Bile salt-induced apoptosis of hepatocytes involves activation of protein kinase C. Am. J. Physiol., 272, (5 Pt 1), 1109-1115.

Jones, D.; Hansen, B. (2016) Editorial: fenofibrate as second-line therapy in high risk PBC--more answers or more questions? Aliment Pharmacol Ther., 43, (5), 648649.

Keppler, D. (2011) Multidrug resistance proteins (MRPs, ABCCs): importance for pathophysiology and drug therapy. Handb. Exp. Pharmacol., (201), 299-323.

Keppler, D. (2014) The roles of MRP2, MRP3, OATP1B1, and OATP1B3 in conjugated hyperbilirubinemia. Drug. Metab. Dispos., 42, (4), 561-565.

Kir, S.; Zhang, Y.; Gerard, R.; Kliewer, S.; Mangelsdorf, D. J. (2012) Nuclear receptors HNF4alpha and LRH-1 cooperate in regulating Cyp7a1 in vivo. $J$. Biol. Chem., 287, (49), 41334-41341.

Kossor, D.; Handler, J.; Dulik, D.; Meunier, P.; Leonard, T.; Goldstein, R. (1993) Cholestatic potentials of alpha-naphthylisothiocyanate (ANIT) and betanaphthylisothiocyanate (BNIT) in the isolated perfused rat liver. Biochem. Pharmacol., 46, (11), 2061-2066.

Kota, B.; Huang, T.; Roufogalis, B. (2005) An overview on biological mechanisms of PPARs. Pharmacol. Res., 51, (2), 85-94.

Kowdley, K.; Luketic, V.; Chapman, R.; Hirschfield, G. ; Poupon, R.; Schramm, C.; Vincent, C.; Rust, C.; Parés, A.; Mason, A.; Marschall, H.; Shapiro, D.; Adorini, L.; Sciacca, C.; Beecher-Jones, T.; Böhm, O.; Pencek, R.; 
Jones, D. (2018) A randomized trial of obeticholic acid monotherapy in patients with primary biliary cholangitis. Hepatology., 67, (5), 1890-1902.

Krahenbuhl, S.; Talos, C.; Fischer, S.; Reichen, J. (1994) Toxicity of bile acids on the electron transport chain of isolated rat liver mitochondria. Hepatology., 19, (2), 471-479.

Kubitz, R.; Droge, C.; Stindt, J.; Weissenberger, K.; Haussinger, D. (2012) The bile salt export pump (BSEP) in health and disease. Clin. Res. Hepatol. Gastroenterol., 36, (6), 536-553.

Lemasters, J.; Nieminen, A.; Qian, T.; Trost, L.; Elmore, S.; Nishimura, Y.; Crowe, R.; Cascio, W.; Bradham, C.; Brenner, D.; Herman, B. (1998) The mitochondrial permeability transition in cell death: a common mechanism in necrosis, apoptosis and autophagy. Biochim. Biophys. Acta., 1366, (1-2), 177196.

Lemasters, J.; Theruvath, T.; Zhong, Z.; Nieminen, A. (2009) Mitochondrial calcium and the permeability transition in cell death. Biochim. Biophys. ActaBioenergetics., 1787, (11), 1395-1401.

Li, T.; Chiang, J. (2009) Regulation of Bile Acid and Cholesterol Metabolism by PPARs. PPAR. Res., doi: 10.1155/2009/501739.

Li, X.; Gong, X.; Zhang, L.; Jiang, R.; Li, H.; Wu, M.; Wan, J. (2013) Protective Effects of Polydatin on Septic Lung Injury in Mice via Upregulation of HO-1. Mediators. Inflamm., doi: 10.1155/2013/354087.

Liu, A.; Wang, W.; Fang, H.; Yang, Y.; Jiang, X.; Liu, S.; Hu, J.; Hu, Q.; Dahmen, U.; Dirsch, O. (2015) Baicalein protects against polymicrobial sepsis-induced liver injury via inhibition of inflammation and apoptosis in mice. Eur. J. Pharmacol., 748, 45-53.

Makishima, M.; Okamoto, A.; Repa, J.; Tu, H.; Learned, R.; Luk, A.; Hull, M.; Lustig, K.; Mangelsdorf, D.; Shan, B. (1999) Identification of a nuclear receptor for bile acids. Science., 284, (5418), 1362-1365.

Makled, M.; El-Awady, M.; Abdelaziz, R.; Atwan, N.; Guns, E.; Gameil, N.; Shehab El-Din, A.; Ammar, E. (2016) Pomegranate protects liver against cecal ligation and puncture-induced oxidative stress and inflammation in rats through TLR/NF-kappaB pathway inhibition. Environ. Toxicol. Pharmacol., 43, 182-192.

Meier, P. (1995) Molecular mechanisms of hepatic bile salt transport from sinusoidal blood into bile. Am. J. Physiol., 269, (6 Pt 1), 801-812.

Meier, P.; Stieger, B. (2002) Bile salt transporters. Annu. Rev. Physiol., 64, 635-661.

Modica, S.; Petruzzelli, M.; Bellafante, E.; Murzilli, S.; Salvatore, L.; Celli, N.; Di Tullio, G.; Palasciano, G.; Moustafa, T.; Halilbasic, E.; Trauner, M.; Moschetta, A. (2012) Selective activation of nuclear bile acid receptor FXR in the intestine protects mice against cholestasis. Gastroenterology., 142, (2), 355-365 e351-354.

Myant, N.; Mitropoulos, K. (1977) Cholesterol 7 alpha-hydroxylase. J. Lipid. Res., 18, (2), 135-153. 
Nagy, L. (2015) The Role of Innate Immunity in Alcoholic Liver Disease. Alcohol. Res., 37, (2), 237-250.

Natalini, B.; Sardella, R.; Gioiello, A.; Ianni, F.; Di Michele, A.; Marinozzi, M. (2014) Determination of bile salt critical micellization concentration on the road to drug discovery. J. Pharm. Biomed. Anal., 87, 62-81.

O'Leary, J.; Pratt, D. (2007) Cholestasis and cholestatic syndromes. Curr. Opin. Gastroenterol., 23, (3), 232-236.

Ortiz, D. F.; Moseley, J.; Calderon, G.; Swift, A.; Li, S.; Arias, I. (2004) Identification of HAX-1 as a protein that binds bile salt export protein and regulates its abundance in the apical membrane of Madin-Darby canine kidney cells. J. Biol. Chem., 279, (31), 32761-32770.

Oude Elferink, R.; Groen, A. (2000) Mechanisms of biliary lipid secretion and their role in lipid homeostasis. Semin. Liver. Dis., 20, (3), 293-305.

Pircher, P.; Kitto, J.; Petrowski, M.; Tangirala, R.; Bischoff, E.; Schulman, I. Westin, S. (2003) Farnesoid $X$ receptor regulates bile acid-amino acid conjugation. J. Biol. Chem., 278, (30), 27703-27711.

Poupon, R. (2012) Ursodeoxycholic acid and bile-acid mimetics as therapeutic agents for cholestatic liver diseases: an overview of their mechanisms of action. $\underline{C l i n}$ Res Hepatol Gastroenterol., 36, (Suppl 1), S3-12.

Poupon, R.; Chazouilleres, O.; Poupon, R. (2000) Chronic cholestatic diseases. $J$. Hepatol., 32, (1 Suppl), 129-140.

Qureshi, W. (1999) Intrahepatic cholestatic syndromes: pathogenesis, clinical features and management. Dig. Dis., 17, (1), 49-59.

Ramaiah, S.; Jaeschke, H. (2007) Role of neutrophils in the pathogenesis of acute inflammatory liver injury. Toxicol. Pathol., 35, (6), 757-766.

Rodrigues, C.; Fan, G.; Wong, P.; Kren, B.; Steer, C. (1998) Ursodeoxycholic acid may inhibit deoxycholic acid-induced apoptosis by modulating mitochondrial transmembrane potential and reactive oxygen species production. Mol. Med., 4, (3), 165-178.

Rodriguez-Garay, E. (2003) Cholestasis: human disease and experimental animal models. Ann. Hepatol., 2, (4), 150-158.

Russell, D.; Setchell, K. (1992) Bile acid biosynthesis. Biochemistry., 31, (20), 47374749.

Scaffidi, P.; Misteli, T.; Bianchi, M. (2010) Release of chromatin protein HMGB1 by necrotic cells triggers inflammation. Nature., 467, (7315), 622-622.

Schubert, R.; Schmidt, K. (1988) Structural changes in vesicle membranes and mixed micelles of various lipid compositions after binding of different bile salts. Biochemistry., 27, (24), 8787-8794.

Stark, G. (2005) Functional consequences of oxidative membrane damage. J. Membr. Biol., 205, (1), 1-16. 
Stieger, B. (2011) The role of the sodium-taurocholate cotransporting polypeptide (NTCP) and of the bile salt export pump (BSEP) in physiology and pathophysiology of bile formation. Handb. Exp. Pharmacol., (201), 205-259.

Susin, S.; Zamzami, N.; Kroemer, G. (1998) Mitochondria as regulators of apoptosis: doubt no more. Biochim. Biophys. Acta., 1366, (1-2), 151-165.

Trauner, M.; Halilbasic, E. (2011) Nuclear receptors as new perspective for the management of liver diseases. Gastroenterology., 140, (4), 1120-1125.

Wada, T.; Gao, J.; Xie, W. (2009) PXR and CAR in energy metabolism. Trends Endocrinol Metab., 20, (6), 273-279.

Wagner, M.; Zollner, G.; Trauner, M. (2011) Nuclear receptors in liver disease. Hepatology., 53, (3), 1023-1034.

Woolbright, B.; Jaeschke, H. (2012) Novel insight into mechanisms of cholestatic liver injury. World. J. Gastroenterol., 18, (36), 4985-4993.

Xu, Z.; Huang, G.; Gong, W.; Zhou, P.; Zhao, Y.; Zhang, Y.; Zeng, Y.; Gao, M.; Pan, Z.; He, F. (2012) FXR ligands protect against hepatocellular inflammation via SOCS3 induction. Cell Signal., 24, (8), 1658-1664.

Yu, L.; Li-Hawkins, J.; Hammer, R.; Berge, K.; Horton, J.; Cohen, J.; Hobbs, H. (2002) Overexpression of ABCG5 and ABCG8 promotes biliary cholesterol secretion and reduces fractional absorption of dietary cholesterol. J. Clin. Invest., 110, (5), 671-680.

Zhang, Y.; Li, S.; He, L.; Wang, F.; Chen, K.; Li, J.; Liu, T.; Zheng, Y.; Wang, J.; Lu, W.; Zhou, Y.; Yin, Q.; Xia, Y.; Zhou, Y.; Lu, J.; Guo, C. (2015) Combination therapy of fenofibrate and ursodeoxycholic acid in patients with primary biliary cirrhosis who respond incompletely to UDCA monotherapy: a meta-analysis. Drug Des Devel Ther., 9, 2757-2766.

Zhao, Q.; Yang, R.; Wang, J.; Hu, D.; Li, F. (2017) PPAR $\alpha$ activation protects against cholestatic liver injury. Scientific Reports., 7, (1), 9967.

Zollner, G.; Trauner, M. (2008) Mechanisms of cholestasis. Clin. Liver. Dis., 12, (1), $1-26$, vii.

Zoratti, M.; Szabo, I. (1995) The mitochondrial permeability transition. Biochim. Biophys. Acta., 1241, (2), 139-176. 
الركود الصفراوي: الآلية الجزيئية ، التطور المرضى والطرق المحتملة للتعامل مع المرض للسادة الدكاترة

بسنت محمود البططي "، أماني على عبسى أحمد"، رحاب كامل محمود"، \#.

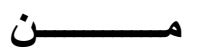

* قسم الأدوية و السموم، كلية الصيدلة، جامعة حلوان، القاهرة، مصر.

"\#م الأدوية والسموم، كلية الصيدلة، جامعة الأهر ام الكندية، القاهرة، مصر.

الخلاصة

إن فهم الآلية الجزيئية لتصنيع و إفراز الصفراء والمسار ات المختلفة لتلك الألية وكذلك التطور المرضى التئ

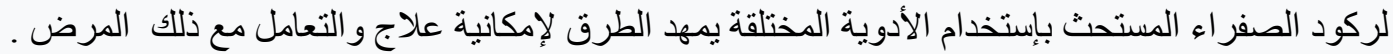

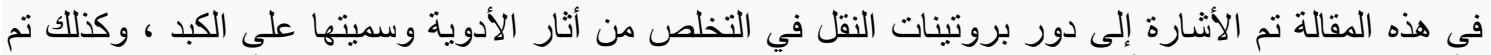

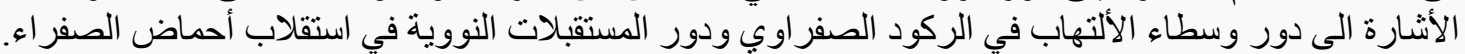

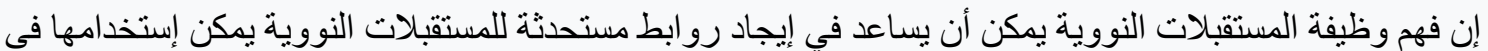
علاج الركود الصفراوي. الكلمات المفتاحية: الأحماض الصفر اوية ، المستقبلات داخل النواة ووسطاء الألتهاب و طرق التعامل مع المرض 